\title{
Interictal quantitative EEG in migraine: a blinded controlled study
}

\author{
Marte Helene Bjørk · Lars J. Stovner • \\ Morten Engstrøm • Marit Stjern · Knut Hagen • \\ Trond Sand
}

Received: 8 May 2009/Accepted: 6 July 2009/Published online: 25 August 2009

(C) Springer-Verlag 2009

\begin{abstract}
Abnormal electroencephalography (EEG) in migraineurs has been reported in several studies. However, few have evaluated EEG findings in migraineurs during a time period when neither the last attack nor the next attack may interact with the results. We, therefore, compared interictal EEG in migraineurs and headache-free subjects with a design controlled for interference by pre-ictal changes. Pre-ictal EEG findings in the painful cranial side during the next attack after registration were also investigated. Correlations between clinical variables and EEG are reported as well. Interictal EEGs from 33 migraineurs (6 with and 27 without aura) and 31 controls were compared. Absolute power, asymmetry and relative power were studied for delta, theta and alpha frequency bands in parieto-occipital, temporal and fronto-central areas. EEG variables were correlated to attack frequency, headache duration, attack duration, pain intensity, photo- and phonophobia. Compared with controls, migraineurs had increased relative theta power in all cortical regions and increased delta activity in the painful fronto-central region. Absolute power and asymmetry were
\end{abstract}

An invited commentary on this article can be found at doi:10.1007/s10194-009-0149-8.

M. H. Bjørk (ه) · L. J. Stovner · M. Stjern · K. Hagen ·

T. Sand

Department of Neuroscience, Norwegian University of Science and Technology (NTNU), MTFS, 7489 Trondheim, Norway

e-mail: marte.bjork@ntnu.no

M. Engstrøm · T. Sand

Department of Neurology and Clinical Neurophysiology,

St. Olavs Hospital, 7006 Trondheim, Norway

L. J. Stovner · M. Stjern · K. Hagen

Norwegian National Headache Centre, St. Olavs Hospital,

7006 Trondheim, Norway similar among groups. In age-adjusted analyses, headache intensity correlated with increased delta activity. In this blinded controlled study, we found globally increased relative theta activity in migraineurs. A slight interictal brain dysfunction is probably present between attacks.

Keywords Migraine $\cdot$ Headache $\cdot$ Hemicrania QEEG $\cdot$ Delta $\cdot$ Theta

\section{Introduction}

Migraine patients are hypersensitive to various stimuli even outside the headache attacks [1-6]. The underlying cause for such symptoms is still unknown. Although positron emission tomography (PET), magnetic resonance imaging (MRI) and blink reflex studies support the presence of a "migraine generator" located in the brainstem [7-9], the presence of visual evoked potentials (VEP) habituation dysfunction [10], transcranial magnetic stimulation threshold changes and somatosensory evoked potential abnormality suggest that there is also a thalamic or cortical dysfunction [11]. This notion of a cortical dysfunction in migraine is also supported by blinded electroencephalography (EEG) studies and controlled quantitative EEG (QEEG) studies showing that EEG abnormality rates are higher in migraineurs compared to headache-free controls [12-15]. However, the results from QEEG studies in migraine are not consistent and partly contradictory [16-19]. The cause for this disagreement has not been clarified, but many QEEG studies did apparently not take into account that some patients were in a pre-attack phase during recording [20-28].

Pre-ictal neurophysiological changes have indeed been found with QEEG [29] as well as with other methods [30-38]. For this reason, it is important to re-evaluate EEG 
findings in migraineurs during a time period when neither the last attack nor the next attack may interact with the results.

Subclinical, possible ischemic, white matter lesions [3943] and grey matter changes [44, 45] have also been described in migraine patients. These findings correlate with attack frequency [40, 44] and disease duration [45]. Because cerebral ischaemia causes increased theta and delta and decreased alpha activity [46-48], it may be interesting to study whether interictal cortical function also correlates with migraine symptoms and severity.

Our aim was accordingly to estimate delta, theta and alpha EEG band power in controls and in migraineurs in a true interictal period, where both the time from the previous attack and the time to the following attack are controlled. We also evaluated interictal EEG findings in the symptomatic hemisphere, and the associations between interictal EEG band power and measures of headache duration, frequency, intensity, phono- and photophobia in migraineurs.

\section{Patients and methods}

Migraine patients were recruited by a newspaper advertisement. After telephone screening by nurses trained in headache research, 52 subjects were examined by a neurologist. The eligible participant was aged 18-65 with 2-6 migraine attacks per month during the previous 3 months. The diagnosis of migraine was made according to the International Headache Society's classification of Headache Disorders 2nd Edition [49], using ICHD-II codes 1.1 (migraine without aura) and 1.2.1 (typical aura with migraine headache). Healthy control subjects were recruited among blood donors. They had a semi-structured interview by an experienced study nurse. Exclusion criteria (migraineurs and controls) were: coexisting frequent episodic or chronic tension-type headache, acute or chronic neurological disease, connective tissue disorder or other painful conditions, malignancy, previous craniotomy or cervical spine surgery, cardiopulmonary or cerebrovascular disease, hypertension, pregnancy, medication for acute or chronic pain, neuroleptics, alcohol or drug abuse, ferromagnetic implants, and use of neuroactive substances such as anti-depressive, anti-epileptic, or migraine prophylactic drugs within 4 weeks before the test. MRI scans were not performed.

Forty-one migraine patients (33 without aura, 8 with aura) and 31 controls were considered for this EEG study. Three EEG recordings were performed in each subject except for two patients who were not willing to undergo all three EEG recordings because headache worsened after the tests. Patients completed a questionnaire about their headache asking about, e.g., disease duration, attack duration, attack frequency (1-3), headache intensity (1-3), and phono/photophobia (0-3). They kept a headache diary for minimum 2 weeks before and after the test, including entries on pain characteristics, accompanying symptoms, consequences for work and leisure, and time of start and end of headache. This enabled a correct retrospective classification of the headache attack and its relationship to the time of the EEG recordings.

Patients without at least one interictal recording (no attack $36 \mathrm{~h}$ before and $36 \mathrm{~h}$ after the recording) were excluded from the analysis. Thirty-six hours cut-off was chosen because it has been shown that extending the time to $72 \mathrm{~h}$ did not influence power values [29]. The diary was incomplete in one patient who had only one EEG. Thirtythree of the remaining 40 patients were accordingly analysed. One of the three EEG recordings was selected from each control subject (day 1, 2, or 3) according to the recording day of a corresponding age-matched patient. Twenty-seven of the 33 patients reported laterality of the attack following the interictal EEG recording. The cranial side reported as most symptomatic during the following attack was selected as the symptomatic side (S). S and "non-symptomatic" (NS) sides were selected by random (either right or left) in controls.

Artefact-free segments were selected for quantitative analysis. An EEG frequency spectrum was obtained with Fast Fourier Transformation (FFT). Definitions and calculations are described in details previously [29]. In brief, we calculated band power values $\left(\mu \mathrm{V}^{2}\right)$ by summing power across all bins in this frequency spectrum for $0.5-3.5 \mathrm{~Hz}$ (delta), 3.75-7.5 Hz (theta) and 7.75-12.5 Hz (alpha). Relative power values were defined as $R=$ band power/ total power. Occipitoparietal (O1, O2, P3, P4), temporal (T3, T4, T4, T6) and fronto-central (F3, F4, C3, C4) regional average values were computed. Absolute asymmetry was calculated as the sign-free difference between the left-sided and the right-sided regional power. In the subgroup of patients with unilateral symptom predominance (hemicrania), we did also calculate the $\mathrm{S}-\mathrm{NS}$ difference (ln-transformed power).

Alpha peak changes and pre-attack EEG band power have been reported previously in paired studies [29, 50]. Other examinations in the 2.5-h long neurophysiological battery were VEP [38], brainstem auditory evoked potentials [37], thermal pain thresholds [30] and pupillary reflex (to be reported in another paper).

The technicians, neurophysiologist, and other staff involved in data reduction and analysis were blinded regarding the diagnostic status. The study was carried out according to the Helsinki declaration. Written consent was obtained from all subjects. They received an amount equivalent to 150 USD after completing the three sessions to cover expenses (not mentioned in the newspaper 
advertisement). The Regional Ethics Committee approved the study.

\section{Statistics}

The analysis was performed with SPSS (version 15.0) and SYSTAT (version 11). We used Fisher exact test for categorical variables. As the distribution of QEEG data was often skewed and some group sizes were small, non-parametric tests were used. The combined (MwoA + MA) migraine group was compared to the control group with Mann-Whitney $U$ tests. In case of significance, we performed a post hoc comparison between MA and MwoA subgroups for the variable in question (Mann-Whitney test). The associations between QEEG (delta, theta, alpha absolute and relative power) and headache history duration, headache attack duration, headache intensity, headache days in the last 3 months, phonophobia, and photophobia were explored with Spearman's rho. Significant associations were then controlled for age with an additional post hoc partial correlation analysis. Band power was ln-transformed before partial correlation analysis. With intention to avoid type II errors, we considered two-sided $p$ values $<0.05$ to be significant.

\section{Results}

Demographic, clinical and EEG quality data are shown in Table 1. There were no significant differences. Groups were also comparable regarding coffee and alcohol use ( $p>0.29, z>-1-1$, Mann-Whitney $U$ test) as well as tobacco smoking ( $p=0.18$, Fisher exact test). Eight of the controls and 12 of the migraine patients used hormonal active medication (birth prevention or replacement therapy, $p=0.43$, Fisher exact test). Three subjects in each group used histamine antagonists $(p=1.0$, Fisher exact test).

Relative theta power was increased in migraine in the parieto-occipital $(p=0.045)$, fronto-central $(p=0.06)$ and temporal region ( $p=0.037$; Table 2; Fig. 1 ). Migraineurs without aura had slightly more relative theta activity than controls in post hoc analysis [mean (SD) in fronto-central area: $0.16(0.04)$ vs. $0.13(0.04), p=0.03$; parieto-occipital area: $0.12(0.04)$ vs. $0.10(0.04), p=0.02$; temporal area: $0.15(0.05)$ vs. $0.12(0.04), p=0.02$; Mann-Whitney $U$ tests]. The other relative power bands as well as absolute power and asymmetry (not tabulated) were similar between groups (Table 2).

Fronto-central delta power was slightly increased on the symptomatic side (Mann-Whitney $U$ test, $p=0.005$; Fig. 2). The increased fronto-central symptomatic-nonsymptomatic delta activity difference was also mainly present in MwoA patients $\left(0.53 \mu \mathrm{V}^{2}\right)$ compared to $-0.03 \mu \mathrm{V}^{2}$ in MA (Mann-Whitney $U$ test, $p=0.29$ ) and $-0.62 \mu \mathrm{V}^{2}$ in controls (Mann-Whitney $U$ test, $p=0.003$ ).

We observed a positive association between headache intensity and delta power (Table 3; Fig. 3) as well as delta asymmetry (fronto-central: rho $=0.38, p=0.03$; parietooccipital: rho $=0.34, p=0.05$; temporal delta asymmetry: rho $=0.52, p=0.001)$. The correlations between fronto-central and temporal delta power and headache intensity were also present after adjustment for age.

Headache history and age were both negatively associated with delta power in migraine patients (Table 3 ). No negative association between age and delta was found among controls (rho $>-0.28, p>0.12$ ), suggesting that headache history duration was a more important predictor for delta power than
Table 1 Demographic and clinical data

MA migraine with aura, MwoA migraine without aura. Eye blinks per minute. Alfa persistence regularity of typical occipital alpha rhythm scored on a six-level scale. Epochs number of 4-s artefact-free EEG epochs included in the spectral analysis

\begin{tabular}{llll}
\hline & $\begin{array}{l}\text { Migraine } \\
(n=33)\end{array}$ & $\begin{array}{l}\text { Migraine with attack } \\
\text { asymmetry }(\mathrm{n}=27)\end{array}$ & $\begin{array}{l}\text { Controls } \\
(n=31)\end{array}$ \\
\hline Women/men & $30 / 3$ & $25 / 2$ & $28 / 3$ \\
MwoA/MA & $27 / 6$ & $21 / 6$ & $40.0(11.4)$ \\
Age (years) & $36.5(12.7)$ & $37.7(12.9)$ & $10.2(9.7)$ \\
Days from last menstruation & $11.0(9.3)$ & $11.4(9.3)$ & \\
Headache history (years) & $19.3(11.0)$ & $20.6(10.3)$ & \\
Headache days last 3 months & $6.2(4.0)$ & $5.9(4.2)$ & \\
Headache intensity (0-4) & $2.4(0.7)$ & $2.5(0.6)$ & $15.7(1.6)$ \\
Headache duration (h) & $17.8(22.0)$ & $18.5(22.9)$ & $5(12)$ \\
Photophobia (0-2) & $1.4(0.7)$ & $1.4(0.7)$ & $2.0(2.8)$ \\
Phonophobia (0-2) & $1.1(0.8)$ & $1.3(0.7)$ & $3.8(1.4)$ \\
EEG epochs & $16.6(2.2)$ & $16.8(2.2)$ & $7(13)$ \\
Percent drowsiness & $7(13)$ & $2.2(4.3)$ & \\
Eye blinks & $2.2(3.9)$ & $4.0(1.5)$ & \\
Alfa persistence (0-5) & $3.8(1.6)$ & &
\end{tabular}


Table 2 Absolute and relative spectral power

\begin{tabular}{|c|c|c|c|c|c|c|}
\hline & \multicolumn{3}{|c|}{ Mean absolute power ${ }^{a}$} & \multicolumn{3}{|c|}{ Mean relative power ${ }^{a}$} \\
\hline & Migraine $(n=33)$ & Controls $(n=31)$ & $p$ & Migraine $(n=33)$ & Controls $(n=31)$ & $p$ \\
\hline \multicolumn{7}{|l|}{ Fronto-central } \\
\hline Delta power $\left(\mu \mathrm{V}^{2}\right)$ & $7.4(3.5)$ & $6.7(2.8)$ & 0.39 & $0.30(0.12)$ & $0.29(0.14)$ & 0.64 \\
\hline Theta power $\left(\mu \mathrm{V}^{2}\right)$ & $4.6(4.2)$ & $3.3(1.8)$ & 0.28 & $0.15(0.04)$ & $0.13(0.04)$ & 0.06 \\
\hline Alpha power $\left(\mu \mathrm{V}^{2}\right)$ & $12.2(12.3)$ & $10.9(9.9)$ & 0.83 & $0.35(0.15)$ & $0.35(0.16)$ & 0.97 \\
\hline \multicolumn{7}{|l|}{ Parieto-occipital } \\
\hline Delta power $\left(\mu \mathrm{V}^{2}\right)$ & $11.7(10.3)$ & $8.5(3.0)$ & 0.29 & $0.22(0.13)$ & $0.20(0.13)$ & 0.46 \\
\hline Theta power $\left(\mu \mathrm{V}^{2}\right)$ & $8.3(11.8)$ & $5.2(3.5)$ & 0.18 & $0.12(0.04)$ & $0.10(0.04)$ & 0.045 \\
\hline Alpha power $\left(\mu \mathrm{V}^{2}\right)$ & $45.8(52.3)$ & $38.6(35.3)$ & 1.00 & $0.50(0.21)$ & $0.51(0.21)$ & 0.73 \\
\hline \multicolumn{7}{|l|}{ Temporal } \\
\hline Delta power $\left(\mu \mathrm{V}^{2}\right)$ & $8.9(6.3)$ & $7.8(3.2)$ & 0.80 & $0.24(0.10)$ & $0.23(0.13)$ & 0.42 \\
\hline Theta power $\left(\mu \mathrm{V}^{2}\right)$ & $6.4(7.0)$ & $4.6(3.0)$ & 0.26 & $0.14(0.05)$ & $0.12(0.04)$ & $\mathbf{0 . 0 3 7}$ \\
\hline Alpha power $\left(\mu \mathrm{V}^{2}\right)$ & $22.0(21.1)$ & $21.2(18.6)$ & 0.95 & $0.43(0.16)$ & $0.43(0.18)$ & 0.84 \\
\hline
\end{tabular}

Bold values represent significant $p$ values $(<0.05)$

$p$ value from non-parametric Mann-Whitney $U$ tests

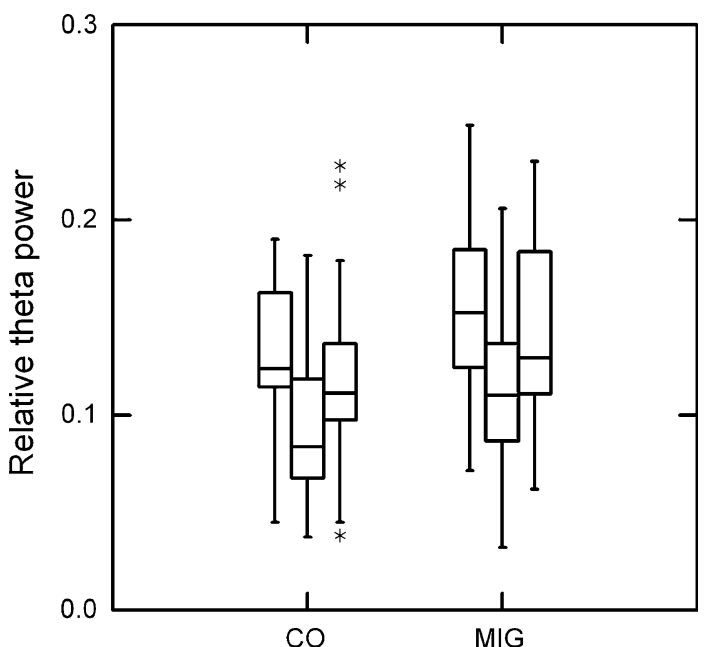

Fig. 1 Interictal relative theta power was increased in migraine in the fronto-central (left box, $p=0.06$ ), parieto-occipital (middle box, $p=0.045$ ) and temporal region (right box, $p=0.037$ ) (MannWhitney $U$ test)

age. However, the relationship between delta power and headache history duration disappeared when age was controlled in partial correlation analysis $(r>-0.24, p>0.17)$. Headache history correlated negatively with delta asymmetry (parieto-occipital: rho $=-0.40, p=0.02$; temporal: rho $=-0.49, \quad p=0.004 ;$ fronto-central: rho $=-0.33$, $p=0.06)$. Age also correlated negatively with parietooccipital (rho $=-0.36, p=0.04$ ) and temporal (rho $=$ $-0.49, p=0.004$ ) delta asymmetry in migraineurs, but not in controls $(r>-0.30, p>0.10)$. After correcting for age, headache history still correlated with parieto-occipital delta asymmetry (log data: partial correlation $r=-0.36$, $p=0.04)$.

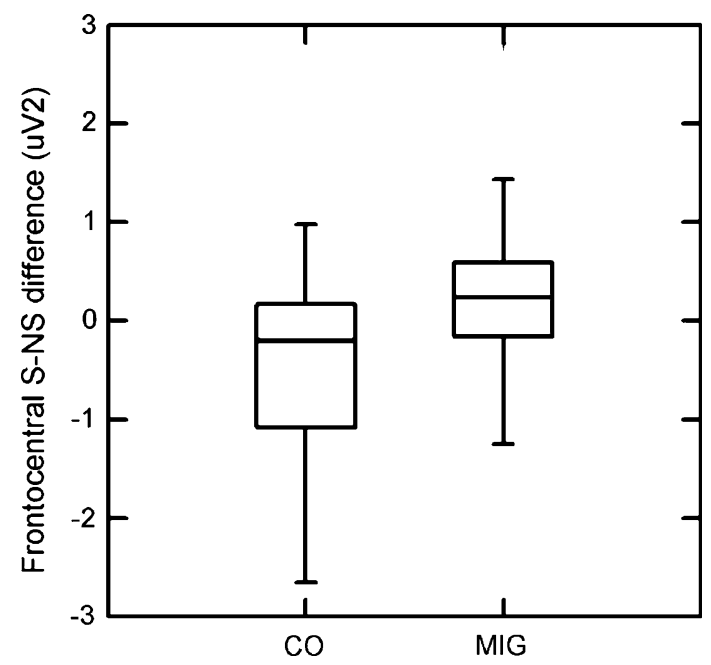

Fig. 2 Larger fronto-central delta power in migraineurs than in controls is observed on the symptomatic side compared to the painfree side ( $\mathrm{S}-\mathrm{NS}$ difference; Mann-Whitney $U$ test, $p=0.005$ ). $\mathrm{S}$ and NS have been selected randomly in the control group. Two outliers are not shown (but included in the statistical analysis)

Photophobia correlated positively with fronto-central absolute theta asymmetry (rho $=0.35, p=0.04$ ), but not after correcting for age.

\section{Discussion}

The main finding in this blinded controlled study was globally increased relative theta activity in migraineurs. This is in accordance with all of the earlier three studies we have come across analyzing interictal relative power in migraineurs. Lia et al. [22] found increased relative theta activity in the parieto-occipital region in adults with and 
Table 3 Correlation between EEG and clinical data in migraine: Spearman rho ( $p$ values)
Bold values represent significant $p$ values $(<0.05)$

* Partial correlation (adjusting for age) was significant $(r>0.36, p<0.05)$. Significant correlations were not found between EEG and headache attack duration, headache days last 3 months, phonophobia or photophobia

\begin{tabular}{lccc}
\hline & Age $($ years $)$ & Headache history (years) & Headache intensity \\
\hline Fronto-central & & & \\
Delta power $\left(\mu \mathrm{V}^{2}\right)$ & $-\mathbf{0 . 5 1}(\mathbf{0 . 0 0 2})$ & $-\mathbf{0 . 4 2}(\mathbf{0 . 0 2})$ & $\mathbf{0 . 5 5}(\mathbf{0 . 0 0 1})^{*}$ \\
Theta power $\left(\mu \mathrm{V}^{2}\right)$ & $-0.13(0.48)$ & $-0.13(0.46)$ & $0.12(0.52)$ \\
Alpha power $\left(\mu \mathrm{V}^{2}\right)$ & $0.16(0.38)$ & $0.11(0.53)$ & $0.16(0.38)$ \\
Parieto-occipital & & & \\
Delta power $\left(\mu \mathrm{V}^{2}\right)$ & $-\mathbf{0 . 5 4}(\mathbf{0 . 0 0 1})$ & $-\mathbf{0 . 4 8}(\mathbf{0 . 0 0 4})$ & $\mathbf{0 . 4 6}(\mathbf{0 . 0 1})$ \\
Theta power $\left(\mu \mathrm{V}^{2}\right)$ & $-0.07(0.71)$ & $-0.08(0.68)$ & $0.07(0.69)$ \\
Alpha power $\left(\mu \mathrm{V}^{2}\right)$ & $0.11(0.53)$ & $0.07(0.71)$ & $0.14(0.43)$ \\
Temporal & & $-\mathbf{0 . 5 4}(\mathbf{0 . 0 0 1})$ & $\mathbf{0 . 5 0}(\mathbf{0 . 0 0 3}) *$ \\
Delta power $\left(\mu \mathrm{V}^{2}\right)$ & $-\mathbf{0 . 5 2}(\mathbf{0 . 0 0 2})$ & $-0.10(0.58)$ & $0.16(0.37)$ \\
Theta power $\left(\mu \mathrm{V}^{2}\right)$ & $-0.07(0.68)$ & $-0.01(0.97)$ & $0.17(0.35)$ \\
Alpha power $\left(\mu \mathrm{V}^{2}\right)$ & $0.04(0.85)$ & $\mathbf{0 . 7 5}(\mathbf{0 . 0 0 0})$ & $-\mathbf{0 . 5 5}(\mathbf{0 . 0 0 1})$ \\
Age & - & $-\mathbf{0 . 4 0}(\mathbf{0 . 0 1})$ & - \\
Headache intensity & $-\mathbf{0 . 5 1}(\mathbf{0 . 0 0 1})$ & & \\
\hline
\end{tabular}

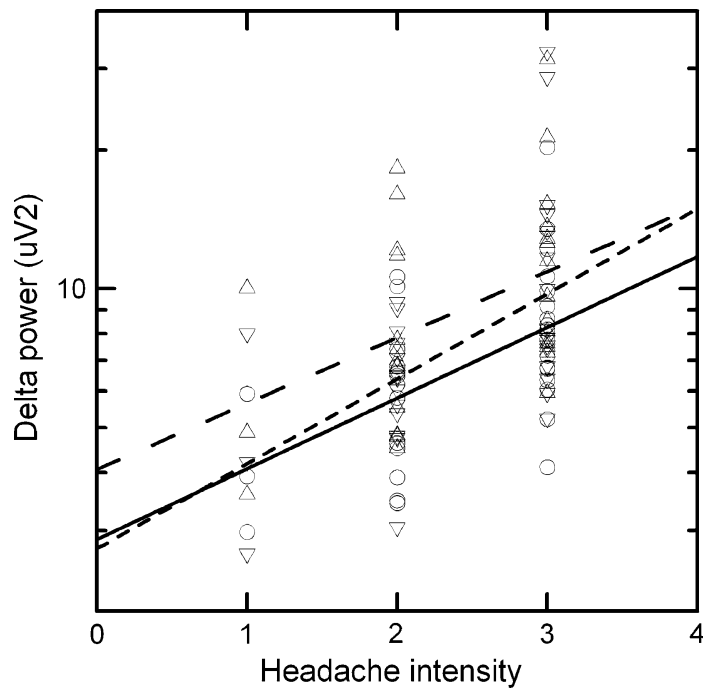

Fig. 3 Linear regression lines are shown for fronto-central (open circles, solid line), parieto-occipital (triangles, broken line) and temporal (inverted triangles, dotted line) delta power (log-transformed $y$-axis)

without aura. Farkas et al. [20] found increased central relative theta power in children with and without aura. Genco et al. [24] reported increased theta, all regions in children with and without aura while only delta was increased in adults.

Generally, older studies have shown slight and inconsistent interictal abnormalities in the alpha and theta bands as reviewed by Sand [17]. We did not find any interictal difference in absolute power values among controls and migraineurs in the present study. These results are supported by a recent, well-designed study [51]. However, an increase of absolute theta activity has previously been found in migraineurs with aura [52]. In a previous paper, we compared EEG power in the interictal period to power in the period before the attack within patients [29]. Interestingly, absolute theta power tended to increase even more just before the following headache attack in the same patients.

Taken together, these findings support a relative excess of slow activity in migraineurs between attacks that possibly increases even more when an attack approaches. We mainly found increased theta activity in migraineurs without aura, but it is plausible that increased theta is also present in migraineurs with aura since our aura subgroup was small and earlier studies have found increased theta in subjects both with and without aura [20, 22, 24].

Theta activity in healthy subjects is linked to hippocampal memory networks during activated behavioural states and thalamic networks during stage 1 NREM sleep [53, 54]. Theta rhythms from the limbic system are generated by subcortical nuclei. Neuronal firing in several other subcortical nuclei including the dorsal raphe nucleus (DRN) and anterior thalamus is phase locked to theta oscillations $[55,56]$. Decreased serotonergic neurotransmission may cause increased theta activities, as serotonergic tone from the DRN normally desynchronizes (suppresses) hippocampal theta. Under experimental conditions, either electrically or chemically lesions or pharmacological depression of DRN have been shown to elicit theta waves [56]. Cholinergic input is important in generating theta activity from the limbic system in awake states [55]. However, during sleep, reduction of either cholinergic, monoaminergic or histaminergic tone from brainstem nuclei to thalamic and cortical neurons is responsible for the transition from faster rhythms towards theta and delta [54, 57].

Pathological theta waves may, on one hand, represent a slowing down of the alpha rhythm due to reduced cerebral blood flow and oxygen uptake in cortical grey matter $[54,58]$. Such slowing is seen in mild to moderate hypoxia, cerebrovascular disease, dementias and mild degrees of 
metabolic encephalopathies. Intermittent, frontotemporal theta, on the other hand, is often attributed to disturbances in deep midline structures [54]. Some believe that theta waves during wakefulness indicate one of several possible pathophysiological conditions, including migraine, collectively termed thalamic dysrhythmias [59].

T- and R-type calcium channels and calcium spikes may be involved in the generation of theta waves $[60,61]$. It can be hypothesized that unstable or hyperactive calciumchannel function is involved in migraine patients, possibly explaining slight cognitive symptoms [62] or dysfunctions in some patients, although genetic studies for some calcium-channel genes have been negative in migraine so far [63, 64].

Another interesting finding was frontal slow delta activity at the side of the head becoming painful during the next headache attack. Asymmetry due to reduction of alpha power with increased or decreased theta power [65] and frequency slowing [66] has been found earlier at the side of the hemicrania during attacks. As power reduction has also been seen 24-48 $\mathrm{h}$ after the attack [65], we cannot exclude an after-effect imposed by the previous attack on the same side. However, we did not find post-ictal QEEG aftereffects in our previous study [29], so this seems less likely. Non-linear sleep EEG analysis has found pre-ictal changes in cortical dynamics at the site of pain maxima of later pain perception [67]. Frontal slow delta may thus reflect unilateral prodromal cortical change in the side that is to be painful, possibly a marker of a latent cortical spreading depression.

Delta activities normally prevail during deep stages of sleep and are most likely generated by cortical, pyramidal neurons between layers II, III and V [54]. It appears when the cholinergic tone from cortically projecting basal forebrain neurons decreases (cortical deafferentation) [54]. T-type calcium channels in thalamocortical networks are also involved in the generation of delta activity during of stage 3 and 4 NREM sleep [61].

We found unilateral slow activity in the frontal lobe. Pathological polymorphic delta activity may occur localized as well as unilateral, and can arise from both metabolic and structural pathologies. Localized delta waves may appear over a subcortical white matter lesion or on the side of thalamic, midbrain reticular formation or hypothalamic lesions [54, 68]. Partial cortical deafferentation may be the cause of this [54]. Reduced frontal grey matter density and diffusion abnormalities have been found by several authors in migraineurs [45, 62, 69]; however, localized lesions of the cortical grey matter do not produce delta activity, only depression of the EEG background activity [68]. In conclusion, slight changes in activity in subcortical structures are probably the most likely substrate of our finding. As MRI scans were not performed, we may not conclude whether cerebral lesions contributed to the findings in this study.

Our main finding in the correlation analysis was an association between headache intensity and increased delta power. In other words, migraineurs with high-intensity attacks have slower activity in their EEG in all cortical areas. The correlation analysis was mainly explorative and hypothesis-generating. Whether our findings are linked to the cause of headache or if it is a consequence of more intense headaches over time remains to be answered. However, a slight cortical dysfunction due to heavy pain may be suspected, as white matter lesions and grey matter changes in migraine patients correlate with severity of migraine $[40,44,45,69,70]$. Cognitive impairments have been found in migraineurs $[62,71]$ correlating with MRI findings [62]. It has been hypothesized that repetitive activation of trigeminovascular neurons and consequently repetitive activation of modulatory pain pathways may lead to impairment of function or partial neuronal cell damage in these areas through the liberation of free radicals [1].

It also seemed like delta activity became more symmetric with increasing age. Asymmetry reduction in older subjects is a well-known phenomenon in functional MRI and PET literature [72]. A compensation theory (agerelated asymmetry reductions might counteract cognitive decline) or a dedifferentiation view (changes reflect a difficulty in recruiting specialized neural mechanisms) is debated [72]. It is, therefore, possible that our results can be explained by a more pronounced cortical "ageing" process in migraineurs compared to controls. It seems like the increased symmetrical activity depends on the disease duration, as headache history also correlated with parietooccipital symmetry after correcting for age. Some authors have found a higher degree of abnormalities in older migraine patients than in younger [28, 52], whereas others found no correlation to clinical severity [25]. In another study (on the same patients), we found that alpha peak frequency slowing also correlated with migraine duration [50]. This may strengthen the assumption that long-term headache affects thalamocortical activity. We did not find correlations with attack frequency, as seen in studies of MRI alterations, but all patients were high frequency migraineurs (between 2 and 6 attacks per month).

Some migraine prophylactics, such as anti-epileptic medication, may influence EEG [73, 74]. Therefore, all neuroactive drugs were ceased at least 4 weeks before EEG recording and did not contribute to the results. Subjects were allowed to use rescue medication, including triptans. However, as all EEGs recorded during attack or within $36 \mathrm{~h}$ before and after migraine attack were excluded, it seems unlikely that these substances could have influenced the results. The half-life of the relevant triptans is between 2 and $6 \mathrm{~h}$. 
Strengths of the present study are the prospective, paired, blinded and controlled design, and the detailed headache diaries completed before and after the tests. We reduced the amount of data and number of comparisons by restricting our computations to three frequency bands and three cortical regions. However, our data must be interpreted with caution, and they need to be replicated before firm conclusions are drawn. Our results are mostly representative for migraineurs without aura and for women, as rather few aura patients and men were recruited. It is debatable whether our $p$ values should be adjusted for multiple comparisons. We have chosen not to do this because Bonferroni-type corrections test the universal null hypotheses (i.e. that all hypotheses are simultaneously nonsignificant). Corrections aimed at preventing type I errors are also associated with increased type II errors [75-77].

In conclusion, in this blinded controlled study, migraineurs had increased relative theta power in a time period free from pre-ictal activity interference. Delta activity was increased even before pain onset in the cranial side to become painful. Patients with high pain intensities had more delta activity than those with less intense pain. These results suggest that migraine is associated with a slight brain dysfunction between attacks, possibly caused by activity changes in subcortical or limbic structures. We underline the need for replication of results before firm conclusions are drawn.

Acknowledgments We are most grateful for the assistance of Grethe Helde, Gøril Bruvik Gravdahl, Nikita Zhitniy. The Norwegian University of Science and Technology (NTNU), The Research Council of Norway and The Norwegian National Headache Centre provided funding.

\section{Conflict of interest None.}

\section{References}

1. Bigal ME, Ferrari M, Silberstein SD, Lipton RB, Goadsby PJ (2009) Migraine in the triptan era: lessons from epidemiology, pathophysiology, and clinical science. Headache 49(Suppl 1):S21-S33

2. Vingen JV, Pareja JA, Storen O, White LR, Stovner LJ (1998) Phonophobia in migraine. Cephalalgia 18(5):243-249

3. Silberstein SD (1995) Migraine symptoms: results of a survey of self-reported migraineurs. Headache 35(7):387-396

4. Vingen JV, Sand T, Stovner LJ (1999) Sensitivity to various stimuli in primary headaches: a questionnaire study. Headache 39(8):552-558

5. Blau JN, Solomon F (1985) Smell and other sensory disturbances in migraine. J Neurol 232(5):275-276

6. Vanagaite J, Pareja JA, Storen O, White LR, Sand T, Stovner LJ (1997) Light-induced discomfort and pain in migraine. Cephalalgia 17(7):733-741

7. Weiller C, May A, Limmroth V, Jüptner M, Kaube H, Schayck $\mathrm{RV}$ et al (1995) Brain stem activation in spontaneous human migraine attacks. Nat Med 1(7):658-660
8. Bahra A, Matharu MS, Buchel C, Frackowiak RS, Goadsby PJ (2001) Brainstem activation specific to migraine headache. Lancet 357(9261):1016-1017

9. Kaube H, Katsarava Z, Przywara S, Drepper J, Ellrich J, Diener HC (2002) Acute migraine headache: possible sensitization of neurons in the spinal trigeminal nucleus? Neurology 58(8):12341238

10. Schoenen J, Wang W, Albert A, Delwaide PJ (1995) Potentiation instead of habituation characterizes visual evoked potentials in migraine patients between attacks. Eur J Neurol 2:115-122

11. Coppola G, Vandenheede M, Di Clemente L, Ambrosini A, Fumal A, De Pasqua V et al (2005) Somatosensory evoked highfrequency oscillations reflecting thalamo-cortical activity are decreased in migraine patients between attacks. Brain $128(\mathrm{Pt}$ 1):98-103

12. Bille BS (1962) Migraine in school children. A study of the incidence and short-term prognosis, and a clinical, psychological and electroencephalographic comparison between children with migraine and matched controls. Acta Paediatr 51(Suppl 136):1151

13. Giel R, de Vlieger M, van Vliet AG (1966) Headache and the EEG. Electroencephalogr Clin Neurophysiol 21(5):492-495

14. Whitehouse D, Pappas JA, Escala PH, Livingston S (1967) Electroencephalographic changes in children with migraine. $\mathrm{N}$ Engl J Med 276(1):23-27

15. Rowan AJ (1974) The electroencephalographic characteristics of migraine. Arch Neurobiol (Madr) 37(Suppl):95-113

16. Gronseth GS, Greenberg MK (1995) The utility of the electroencephalogram in the evaluation of patients presenting with headache: a review of the literature. Neurology 45(7):1263-1267

17. Sand T (1991) EEG in migraine: a review of the literature. Funct Neurol 6(1):7-22

18. Schoenen J (1992) Clinical neurophysiology studies in headache: a review of data and pathophysiological hints. Funct Neurol 40(4):191-204

19. Sand T (2003) Electroencephalography in migraine: a review with focus on quantitative electroencephalography and the migraine vs. epilepsy relationship. Cephalalgia 23(Suppl 1):5-11

20. Farkas V, Benninger C, Matthis P, Scheffner D, Lindeisz F (1987) The EEG background activity in children with migraine. Cephalalgia 6:59-64

21. Neufeld MY, Treves TA, Korczyn AD (1991) EEG and topographic frequency analysis in common and classic migraine. Headache 31(4):232-236

22. Lia C, Carenini L, Degioz C, Bottachi E (1995) Computerized EEG analysis in migraine patients. Ital J Neurol Sci 16(4):249-254

23. Bramanti P, Grugno R, Vitetta A, Di Bella P, Muscara N, Nappi G (2005) Migraine with and without aura: electrophysiological and functional neuroimaging evidence. Funct Neurol 20(1):29-32

24. Genco S, de Tommaso M, Prudenzano AM, Savarese M, Puca FM (1994) EEG features in juvenile migraine: topographic analysis of spontaneous and visual evoked brain electrical activity: a comparison with adult migraine. Cephalalgia 14(1):41-46

25. Jonkman EJ, Lelieveld MH (1981) EEG computer analysis in patients with migraine. Electroencephalogr Clin Neurophysiol 52(6):652-655

26. Rainero I, Amanzio M, Vighetti S, Bergamasco B, Pinessi L, Benedetti F (2001) Quantitative EEG responses to ischaemic arm stress in migraine. Cephalalgia 21(3):224-229

27. Vonderheid-Guth B, Todorova A, Wedekind W, Dimpfel W (2000) Evidence for neuronal dysfunction in migraine: concurrence between specific qEEG findings and clinical drug response-a retrospective analysis. Eur J Med Res 5(11):473-483

28. Facchetti D, Marsile C, Faggi L, Donati E, Kokodoko A, Poloni M (1990) Cerebral mapping in subjects suffering from migraine with aura. Cephalalgia 10(6):279-284 
29. Bjork M, Sand T (2008) Quantitative EEG power and asymmetry increase $36 \mathrm{~h}$ before a migraine attack. Cephalalgia 28(9):960968

30. Sand T, Zhitniy N, Nilsen KB, Helde G, Hagen K, Stovner LJ (2008) Thermal pain thresholds are decreased in the migraine preattack phase. Eur J Neurol 15(11):1199-1205

31. Sand T, Vingen JV (2000) Visual, long-latency auditory and brainstem auditory evoked potentials in migraine: relation to pattern size, stimulus intensity, sound and light discomfort thresholds and pre-attack state. Cephalalgia 20(9):804-820

32. Judit A, Sandor PS, Schoenen J (2000) Habituation of visual and intensity dependence of auditory evoked cortical potentials tends to normalize just before and during the migraine attack. Cephalalgia 20(8):714-719

33. Fritzer G, Strenge H, Goder R, Gerber WD, Aldenhoff J (2004) Changes in cortical dynamics in the preictal stage of a migraine attack. J Clin Neurophysiol 21(2):99-104

34. Kropp P, Gerber WD (1998) Prediction of migraine attacks using a slow cortical potential, the contingent negative variation. Neurosci Lett 257(2):73-76

35. Siniatchkin M, Gerber WD, Kropp P, Vein A (1999) How the brain anticipates an attack: a study of neurophysiological periodicity in migraine. Funct Neurol 14(2):69-77

36. Bowyer SM, Aurora KS, Moran JE, Tepley N, Welch KM (2001) Magnetoencephalographic fields from patients with spontaneous and induced migraine aura. Ann Neurol 50(5):582-587

37. Sand T, Zhitniy N, White LR, Stovner LJ (2008) Brainstem auditory-evoked potential habituation and intensity-dependence related to serotonin metabolism in migraine: a longitudinal study. Clin Neurophysiol 119(5):1190-1200

38. Sand T, Zhitniy N, White LR, Stovner LJ (2008) Visual evoked potential latency, amplitude and habituation in migraine: a longitudinal study. Clin Neurophysiol 119(5):1020-1027

39. Kruit MC, Launer LJ, van Buchem MA, Terwindt GM, Ferrari MD (2005) MRI findings in migraine. Rev Neurol 128(Pt 9):661665

40. Kruit MC, van Buchem MA, Hofman PA, Bakkers JT, Terwindt GM, Ferrari MD et al (2004) Migraine as a risk factor for subclinical brain lesions. JAMA 291(4):427-434

41. Kruit MC, Launer LJ, Ferrari MD, van Buchem MA (2006) Brain stem and cerebellar hyperintense lesions in migraine. Stroke 37(4):1109-1112

42. Kruit MC, Launer LJ, Ferrari MD, van Buchem MA (2005) Infarcts in the posterior circulation territory in migraine. The population-based MRI CAMERA study. Brain 128(Pt 9):20682077

43. Rozen TD (2007) Vanishing cerebellar infarcts in a migraine patient. Cephalalgia 27(6):557-560

44. Valfre W, Rainero I, Bergui M, Pinessi L (2008) Voxel-based morphometry reveals gray matter abnormalities in migraine. Headache 48(1):109-117

45. Rocca MA, Ceccarelli A, Falini A, Colombo B, Tortorella P, Bernasconi L et al (2006) Brain gray matter changes in migraine patients with T2-visible lesions: a 3-T MRI study. Stroke 37(7):1765-1770

46. Finnigan SP, Walsh M, Rose SE, Chalk JB (2007) Quantitative EEG indices of sub-acute ischaemic stroke correlate with clinical outcomes. Clin Neurophysiol 118(11):2525-2532

47. Cohen BA, Bravo-Fernandez EJ, Sances A Jr (1976) Quantification of computer analyzed serial EEGs from stroke patients. Electroencephalogr Clin Neurophysiol 41(4):379-386

48. Nuwer MR, Jordan SE, Ahn SS (1987) Evaluation of stroke using EEG frequency analysis and topographic mapping. Neurology 37(7):1153-1159

49. Headache Classification Committee of the International Headache Society (2004) Classification and diagnostic criteria for headache disorders, cranial neuralgias and facial pain. Cephalalgia 24(Suppl 1):1-160

50. Bjork MH, Stovner LJ, Nilsen BM, Stjern M, Hagen K, Sand T (2009) The occipital alpha rhythm related to the "migraine cycle" and headache burden: a blinded, controlled longitudinal study. Clin Neurophysiol 120(3):464-471

51. Clemens B, Bank J, Piros P, Bessenyei M, Veto S, Toth $\mathrm{M}$ et al (2008) Three-dimensional localization of abnormal EEG activity in migraine: a low resolution electromagnetic tomography (LORETA) study of migraine patients in the pain-free interval. Brain Topogr 21(1):36-42

52. Hughes JR, Robbins LD (1990) Brain mapping in migraine. Clin Electroencephalogr 21(1):14-24

53. Klimesch W, Freunberger R, Sauseng P, Gruber W (2008) A short review of slow phase synchronization and memory: evidence for control processes in different memory systems? Brain Res 1235:31-44

54. Steriade M, Gloor P, Linas R, Lopes da Silva F, Mesulam MM (1990) Report of IFCN Committee on Basic Mechanisms. Basic mechanisms of cerebral rhythmic activities. Electroencephalogr Clin Neurophysiol 76(76):481-508

55. Buzsaki G (2002) Theta oscillations in the hippocampus. Neuron 33(3):325-340

56. Vertes RP, Kocsis B (1997) Brainstem-diencephalo-septohippocampal systems controlling the theta rhythm of the hippocampus. Neuroscience 81(4):893-926

57. McCormick DA (1992) Neurotransmitter actions in the thalamus and cerebral cortex and their role in neuromodulation of thalamocortical activity. Prog Neurobiol 39(4):337-388

58. Ingvar DH, Sjolund B, Ardo A (1976) Correlation between dominant EEG frequency, cerebral oxygen uptake and blood flow. Electroencephalogr Clin Neurophysiol 41(3):268-276

59. Llinas RR, Ribary U, Jeanmonod D, Kronberg E, Mitra PP (1999) Thalamocortical dysrhythmia: a neurological and neuropsychiatric syndrome characterized by magnetoencephalography. Proc Natl Acad Sci USA 96(26):15222-15227

60. Tai C, Kuzmiski JB, MacVicar BA (2006) Muscarinic enhancement of R-type calcium currents in hippocampal CA1 pyramidal neurons. J Neurosci 26(23):6249-6258

61. Crunelli V, Cope DW, Hughes SW (2006) Thalamic T-type $\mathrm{Ca} 2+$ channels and NREM sleep. Cell Calcium 40(2):175-190

62. Schmitz N, Arkink EB, Mulder M, Rubia K, Admiraal-Behloul F, Schoonman GG et al (2008) Frontal lobe structure and executive function in migraine patients. Neurosci Lett 440(2):92-96

63. Thomsen LL, Oestergaard E, Bjornsson A, Stefansson H, Fasquel AC, Gulcher J et al (2008) Screen for CACNA1A and ATP1A2 mutations in sporadic hemiplegic migraine patients. Cephalalgia 28(9):914-921

64. Jen JC, Kim GW, Dudding KA, Baloh RW (2004) No mutations in CACNA1A and ATP1A2 in probands with common types of migraine. Arch Neurol 61(6):926-928

65. Schoenen J, Jamart B, Delwaide PJ (1987) Cartographie electroencephalographique dans les migraines en periodes critique et intercritique. Rev Electroencephalogr Neurophysiol Clin 17(3):289-299

66. de Tommaso M, Sciruicchio V, Guido M, Sasanelli G, Specchio LM, Puca FM (1998) EEG spectral analysis in migraine without aura attacks. Cephalalgia 18(6):324-328

67. Fritzer G, Strenge H, Göder R, Gerber WD, Aldenhoff J (2004) Changes in cortical dynamics in the preictal stage of a migraine attack. J Clin Neurophysiol 21(2):99-104

68. Gloor P, Ball G, Schaul N (1977) Brain lesions that produce delta waves in the EEG. Neurology 27(4):326-333

69. Rocca MA, Ceccarelli A, Falini A, Tortorella P, Colombo B, Pagani E et al (2006) Diffusion tensor magnetic resonance imaging at 3.0 $\mathrm{T}$ shows subtle cerebral grey matter abnormalities 
in patients with migraine. J Neurol Neurosurg Psychiatry 77(5):686-689

70. Schmitz N, Admiraal-Behloul F, Arkink EB, Kruit MC, Schoonman GG, Ferrari MD et al (2008) Attack frequency and disease duration as indicators for brain damage in migraine. Headache 48(7):1044-1055

71. Waldie KE, Hausmann M, Milne BJ, Poulton R (2002) Migraine and cognitive function: a life-course study. Neurology 59(6):904-908

72. Cabeza R (2002) Hemispheric asymmetry reduction in older adults: the HAROLD model. Psychol Aging 17(1):85-100

73. Salinsky MC, Oken BS, Storzbach D, Dodrill CB (2003) Assessment of CNS effects of antiepileptic drugs by using quantitative EEG measures. Epilepsia 44(8):1042-1050
74. Clemens B, Menes A, Piros P, Bessenyei M, Altmann A, Jerney J et al (2006) Quantitative EEG effects of carbamazepine, oxcarbazepine, valproate, lamotrigine, and possible clinical relevance of the findings. Epilepsy Res 70(2-3):190-199

75. Perneger TV (1998) What's wrong with Bonferroni adjustments. BMJ 316(7139):1236-1238

76. Feise RJ (2002) Do multiple outcome measures require $p$ value adjustment? BMC Med Res Methodol 2:8

77. Schulz KF, Grimes DA (2005) Multiplicity in randomised trials I: endpoints and treatments. Lancet 365(9470):1591-1595 